\title{
Uso de la metodología de aprendizaje basada en proyectos para la adquisición de competencias transversales con niveles de dominio alto en asignaturas relacionadas con la Bioelectricidad
}

\author{
Beatriz Trenor ${ }^{\mathrm{a}}$, Gema Prats ${ }^{\mathrm{a}}$ \\ ${ }^{a}$ Departamento de Ingeniería Electrónica, Universitat Politècnica de València. btrenor@eln.upv.es,
} geprabo@eln.upv.es.

\begin{abstract}
The the need for greater participation and motivation of university students in the teachinglearning process has led to the concept of project-based learning. By using this methodology, the student works individually and in groups independently, but under the guidance of the teacher. Furthermore, this methodology allows the acquisition of transversal skills with a high level of mastery in subjects of the last year of undergraduate and master degrees. The objective of this work is the design and application of the project-based learning methodology in the subjects of Bioelectronics and Modeling and simulation of bioelectric systems that is capable of integrating the transversal competences "Design and project" (CT05), "Effective communication "(CT08), "Understanding and integration "(CT01)," Analysis and problem solving "(CT03), "Innovation, creativity and entrepreneurship "(CT04) and" Critical thinking "(CT09), with high levels of mastery. This methodology has been applied throughout the 2019-2020 academic year in these subjects belonging to the Degree in Industrial Electronic and Automatic Engineering and the Master of Biomedical Engineering, respectively, from the Universitat Politècnica de València. The assessment of the students has been very positive and the acquisition of transversal skills has been very satisfactory.
\end{abstract}

Keywords: Transversal skills, Project-based learning, Group work, Rubrics

\section{Resumen}

De la necesidad de una mayor participación y motivación del alumnado universitario en el proceso de enseñanza-aprendizaje ha surgido el concepto de aprendizaje basado en proyectos. Mediante el uso de esta metodología, el alumno trabaja, de manera individual y grupal de manera independiente pero bajo la guía del profesor. Además, esta metodología permite la adquisición de competencias transversales con un alto nivel de dominio en asignaturas de último curso de titulaciones grado y de máster. El objetivo del presente trabajo es el diseño y aplicación de la metodología de aprendizaje basada en proyectos en las asignaturas de Bioelectrónica y Modeling and simulation of bioelectric systems que sea capaz de integrar las competencias transversales "Diseño y proyecto" (CT05), "Comunicación efectiva" (CT08), "Compresión e integración" (CT01), “Análisis y resolución de problemas" (CT03), "Innovación, creatividad y emprendimiento" (CT04) y "Pensamiento crítico" (CT09), con altos niveles de dominio. Se ha aplicado esta metodología a lo largo del curso 2019-2020 en dichas asignaturas pertenecientes al Grado de Ingeniería Electrónica Industrial y Automática y el Máster de Ingeniería Biomédica, respectivamente de la Universitat Politècnica de València. La valoración del alumnado ha sido muy positiva y la adquisición de competencias transversales ha sido muy satisfactoria. 
Palabras clave: Competencias Transversales, Aprendizaje basado en proyectos, Trabajo en grupo, Rúbricas.

\section{Introducción}

El presente trabajo de innovación docente se enmarca en dos asignaturas optativas relacionadas con la bioelectricidad impartidas en distintas titulaciones. Estas asignaturas son Biolectrónica (12194), asignatura de 4.5 créditos impartida en el cuatrimestre B de cuarto curso en el Grado en Ingeniería Electrónica Industrial y Automática y la asignatura Modeling and Simulation of Bioelectric Systems (34374) de 4.5 créditos impartida en el cuatrimestre B en el Máster Universitario en Ingeniería Biomédica. El número de alumnos oscila entre 8 y 20 para Bioelectrónica, y entre 25 y 40 para Modeling and Simulation of Bioelectric Systems.

Los alumnos presentan un alto grado de motivación por los contenidos de las asignaturas, lo cual facilita la implantación de metodologías activas, dentro del contexto del EEES que supone un modelo de aprendizaje centrado en el alumno. Como describe Zabalza (2011), el profundo cambio que ha supuesto el proceso de Bolonia en el aprendizaje de los estudiantes universitarios nos ha permitido pasar del "instruction paradigm" al "learning facilitation paradigm". Así pues mediante metodologías activas pretendemos que los alumnos adquieran las competencias transversales con niveles de dominio alto y que el proceso de aprendizaje sea lo más eficiente y motivador posible.

La Universitat Politècnica de València (UPV) ha implantado un proyecto institucional con trece competencias transversales (CTs) para formar y acreditar a los estudiantes egresados en cualquiera de los títulos oficiales impartidos. Las CTs se trabajan en las distintas asignaturas, y se lleva a cabo el seguimiento del progreso de los estudiantes a través de asignaturas seleccionadas como puntos de control. Los profesores pretendemos diseñar una metodología de enseñanza para optimizar el proceso de aprendizaje del alumno tanto de las competencias genéricas y específicas de la titulación como de las competencias transversaes. En la asignatura Bioelectrónica, las competencias transversales punto de control son: CT05 Diseño y proyecto y CT08 Comunicación efectiva, en las que se prentende alcanzar un nivel de dominio II. En la asignatura Modeling and Simulation of Bioelectric Systems las competencias transversales son: CT01 Compresión e integración, CT03 Análisis y resolución de problemas, CT04 Innovación, creatividad y emprendimiento y CT09 Pensamiento crítico, en las que se pretende alcanzar un nivel de dominio III.

La metodología de aprendizaje basada en proyectos (ABP) reúne las características adecuadas para poder integrar las competencias transversales anteriormente mencionadas en el proceso de aprendizajeenseñanza. En efecto, de acuerdo con Tippelt y Lindemann (2001), el aprendizaje basado en proyectos permite integrar durante las distintas fases de desarrollo del proyecto competencias específicas como la integración del conocimiento, competencias metodológicas como planificación y diseño y competencias humanas y sociales como la comunicación y la creatividad. Por otra parte, puesto que los alumnos de estas asignaturas son alumnos último curso de grado y de máster, respectivamente, las metodologías diseñadas deben permitir alcanzar niveles superiores de la taxonomía de objetivos de Bloom (Fernández 2006). En concreto, el aprendizaje basado en proyectos alcanza un alto nivel de objetivos cognitivos y tiene la capacidad de propiciar un aprendizaje autónomo y continuo donde el estudiante posee un alto grado de control de su aprendizaje.

En cuanto al número de estudiantes de las dos asignaturas es bajo o medio y por tanto el uso de esta metodología es viable para estos grupos y no requiere un número elevado de horas de preparación, de encuentros y correcciones (Fernández 2006). Además, los profesores implicados tienen experiencia en la 
aplicación de innovaciones docente tanto en pequeños grupos (Trenor y Prats-Boluda, 2017) como en grandes grupos (Prats-Boluda et al., 2017; Ye Lin et al., 2017; Ye Lin et al., 2018; Ye Lin et al, 2019; Prats-Boluda et al., 2016), como se indica en la última sección del documento. Las dos asignaturas poseen características similares en cuanto al tema (bioelectricidad), motivación de los alumnos, grupos reducidos, y por ello, en el presente trabajo se plantea el diseño de una metodología común para ambas.

\section{Objetivos}

El objetivo general del presente estudio es el diseño y aplicación de la metodología de aprendizaje basada en proyectos en las asignaturas de Bioelectrónica y Modeling and simulation of bioelectric systems que sea capaz de integrar las competencias transversales "Diseño y proyecto" (CT05), "Comunicación efectiva" (CT08), "Compresión e integración" (CT01), "Análisis y resolución de problemas" (CT03), "Innovación, creatividad y emprendimiento" (CT04) y "Pensamiento crítico" (CT09), con altos niveles de dominio.

Para la consecución del objetivo general, se han planteado los siguientes objetivos específicos:

- Rediseñar los contenidos y la programación de las 2 asignaturas para poder introducir una metodología común de aprendizaje basado en proyectos.

- Implantar distintos tipos de proyectos relacionados con la metodología de aprendizaje basada en proyectos.

- Definir las estrategias de seguimiento y evaluación de los distintos tipos de proyectos realizados por los alumnos.

- Diseñar rúbricas para evaluar las competencias transversales involucradas en el proceso de aprendizaje basado en proyectos.

- Analizar la satisfacción del profesorado y estudiantes y el logro de los objetivos del proyecto.

- Analizar la sostenibilidad de la metodología basada en proyectos analizando tanto el tiempo de dedicación del alumnado como el del profesorado.

- Difundir los resultados de la experiencia realizada en congresos y/o revistas especializados.

\section{Desarrollo de la innovación}

Para la ejecución del presente estudio se propusieron las fases y tareas descritas a continuación y resumidas en el cronograma de la Tabla 1.

La primera fase del trabajo fue la fase de diseño durante el primer cuatrimestre del curso 2019/2020. En esta fase, como primera tarea se seleccionaron los contenidos de las asignaturas, distribuyéndolos en las sesiones de teoría, prácticas y proyectos. La segunda tarea fue el diseño detallado de la metodología: lecciones magistrales participativas, prácticas de laboratorio y aprendizaje basado en proyectos. La tercera tarea fue la definición detallada de los proyectos, considerando 2 proyectos distintos en cada asignatura para cada grupo de alumnos. Estas ideas se plasmaron en documentos concretos como quinta tarea. La sexta tarea fue la integración de las competencias transversales en los distintos proyectos de manera que los alumnos alcancen un alto nivel de dominio. La séptima tarea de esta fase fue el diseño de los métodos de evaluación de los proyectos incluyendo rúbricas, reuniones, correcciones, etc. En la octava tarea se diseñó el método de evaluación completa de la asignatura con detalle. Finalmente, última 
tarea de esta fase fue la previsión de horas de trabajo que tanto alumnos como profesores dedicaron para la implantación de la metodología de aprendizaje basada en proyectos.

La segunda fase del estudio fue la implantación de la metodología de aprendizaje basado en proyectos y tendrá lugar durante el cuatrimestre B del curso 2019-2020, cuatrimestre en el cual se han impartido las asignaturas de Bioelectrónica y Modeling and simulation of bioelectric systems. La primera tarea de esta fase fue la explicación en clase a los alumnos de las metodologías empleadas en la asignatura, haciendo especial hincapié en el aprendizaje basado en proyectos. Durante las primeras semanas de clase, la tarea dos fue el seguimiento del aprendizaje basado en proyectos que incluía el pase de una encuesta inicial para recoger evidencias y evaluar la progresión, tutorías, correcciones, etc. La tercera tarea fue la evaluación final de los proyectos y la cuarta tarea la evaluación del nivel de dominio de las competencias transversales. La cuarta tarea fue la evaluación final de la asignatura, para finalizar con la quinta tarea en la que se diseñó y pasó una encuesta para evaluar la metodología empleada.

Finalmente, durante la fase 3 del proyecto, al finalizar la asignatura se realizó la evaluación de los resultados, cuya primera tarea fue el análisis y valoración del presente trabajo de innovación docente y la segunda tarea la publicación y difusión de los resultados.

La primera fase del trabajo fue la fase de diseño durante el primer cuatrimestre del curso 2019/2020. En esta fase, como primera tarea se seleccionaron los contenidos de las asignaturas, distribuyéndolos en las sesiones de teoría, prácticas y proyectos. La segunda tarea fue el diseño detallado de la metodología: lecciones magistrales participativas, prácticas de laboratorio y aprendizaje basado en proyectos. La tercera tarea fue la definición detallada de los proyectos, considerando 2 proyectos distintos en cada asignatura para cada grupo de alumnos. Estas ideas se plasmaron en documentos concretos como quinta tarea. La sexta tarea fue la integración de las competencias transversales en los distintos proyectos de manera que los alumnos alcancen un alto nivel de dominio. La séptima tarea de esta fase fue el diseño de los métodos de evaluación de los proyectos incluyendo rúbricas, reuniones, correcciones, etc. En la octava tarea se diseñó el método de evaluación completa de la asignatura con detalle. Finalmente, última tarea de esta fase fue la previsión de horas de trabajo que tanto alumnos como profesores dedicaron para la implantación de la metodología de aprendizaje basada en proyectos.

La segunda fase del estudio fue la implantación de la metodología de aprendizaje basado en proyectos y tendrá lugar durante el cuatrimestre B del curso 2019-2020, cuatrimestre en el cual se han impartido las asignaturas de Bioelectrónica y Modeling and simulation of bioelectric systems. La primera tarea de esta fase fue la explicación en clase a los alumnos de las metodologías empleadas en la asignatura, haciendo especial hincapié en el aprendizaje basado en proyectos. Durante las primeras semanas de clase, la tarea dos fue el seguimiento del aprendizaje basado en proyectos que incluía el pase de una encuesta inicial para recoger evidencias y evaluar la progresión, tutorías, correcciones, etc. La tercera tarea fue la evaluación final de los proyectos y la cuarta tarea la evaluación del nivel de dominio de las competencias transversales. La cuarta tarea fue la evaluación final de la asignatura, para finalizar con la quinta tarea en la que se diseñó y pasó una encuesta para evaluar la metodología empleada.

Finalmente, durante la fase 3 del proyecto, al finalizar la asignatura se realizó la evaluación de los resultados, cuya primera tarea fue el análisis y valoración del presente trabajo de innovación docente y la segunda tarea la publicación y difusión de los resultados. 
Tabla 1. Cronograma del pan de trabajo. Responsables de las tareas: Beatriz Trénor (BT), Gema Prats (GP), Lucía Romero (LR), Javier Saiz (JS), José Maria Ferrero (JMF).

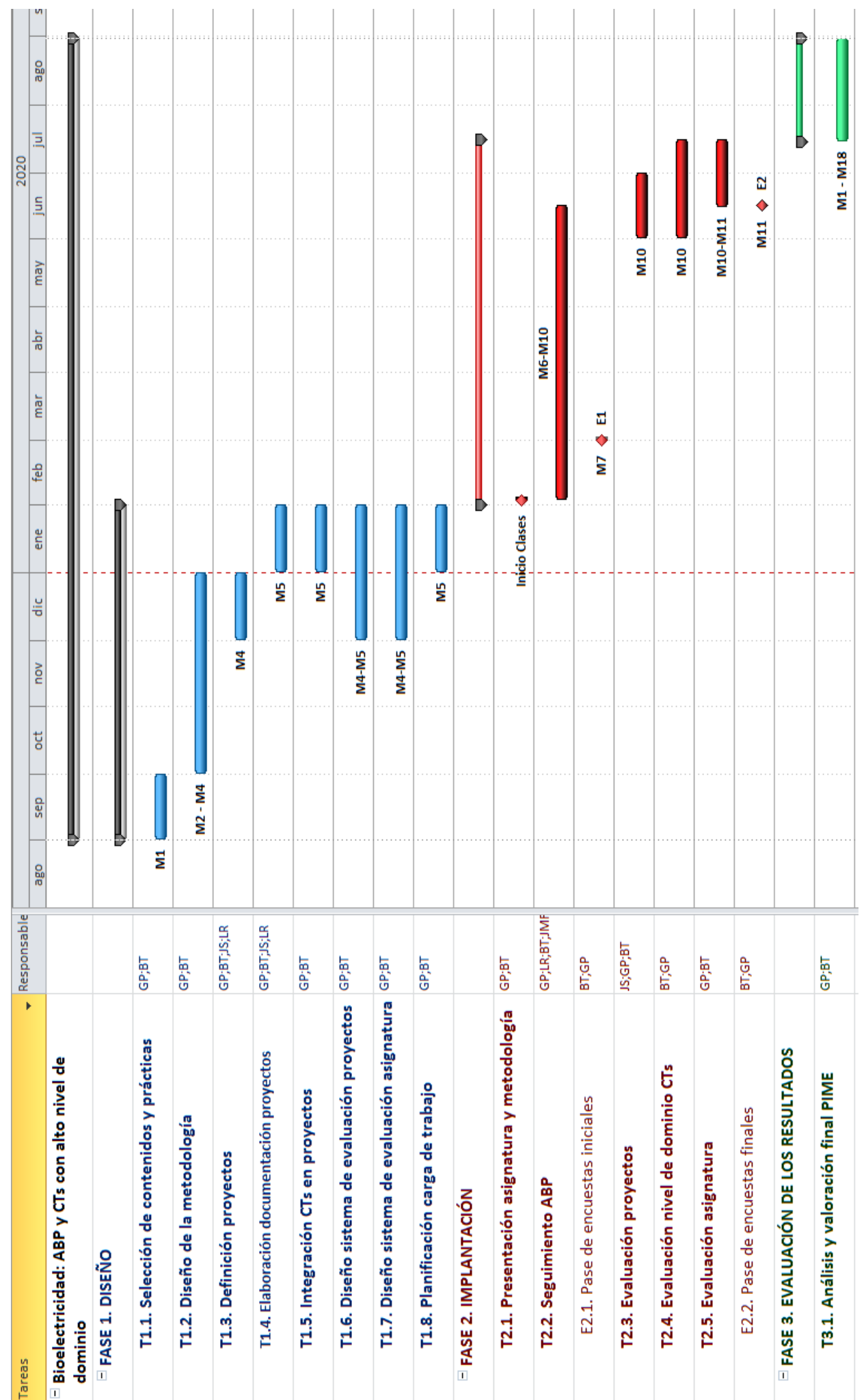




\section{Resultados}

Para evaluar el logro alcanzado de los objetivos planteados se diseñó con detalle durante la primera fase del proyecto una serie de encuestas y rúbricas que nos permitieron a los profesores evidenciar el resultado obtenido.

Concretamente, en la primera fase de los distintos proyectos y tras su finalización se pasaron encuestas a los alumnos en las que se preguntó acerca del tiempo dedicado y el nivel de dominio de las competencias concretas. Estas encuestas y sus resultados se muestran en las tablas 2 a 5 para la asignatura de Modeling and simulation of bioelectric systems y en las tablas 6 y 7 para la asignatura de Bioelectróncia. De este modo se ha podido medir la progresión en la adquisición de las competencias. Además, se ha medido la viabilidad del proyecto y si el tiempo dedicado entra dentro de los límites definidos por los ECTS de las asignaturas.

Según las encuestas realizadas a los alumnos de la asignatura de Modeling and simulation of bioelectric systems (tabla 2), éstos no son inicialmente conocedores de las competencias transversales que se trabajan en la asignatura. Además, al finalizar la asignatura reconocen haber alcanzado un nivel alto en las competencias CT01 y CT09. Consideran que el análisis del artículo científico les ha ayudado a la comprensión e integración de los contenidos de la asignatura y han potenciado su pensamiento crítico.

Los alumnos han realizado una planificación temporal inicial que han cumplido durante el desarrollo del trabajo, han trabajado en grupo de manera adecuada y consideran que el trabajo les ha ayudado en gran medida a afianzar los conceptos vistos en la asignatura, y están satisfechos con su realización (tabla 3).

Con respecto al trabajo de simulación (tablas 4 y 5), los alumnos consideran que su realización ha contribuido favorablemente a la adquisición de un nivel alto en las competencias CT03 y CT04. Los alumnos han mejorado a lo largo del desarrollo del proyecto en el análisis y resolución de problemas con profundidad y de manera sistemática. Asimismo consideran que han demostrado cualidades de creatividad durante el desarrollo del trabajo. La mayoría de los alumnos han dedicado el tiempo previsto al trabajo, aunque algunos consideran que han dedicado algo más de tiempo. Han trabajado en equipo de manera muy adecuada y consideran que el desarrollo del proyecto ha sido muy beneficioso.

Tabla 2. Encuesta inicial y final sobre la adquisición de competencias transversales durante el análisis de un artículo cientifico en la asignatura de Moldeling and simulation of bioelectric systems.

\begin{tabular}{|c|c|c|}
\hline Preguntas Competencias. Análisis de Artículo científico n=19 & Respuesta Inicial & Respuesta Final \\
\hline $\begin{array}{l}\text { 1. ¿Conoces el programa de competencias transversales de la UPV? } \\
\text { A. No } \\
\text { B. Muy poco } \\
\text { C. Bien } \\
\text { D. Muy bien }\end{array}$ & $=\mathrm{A}=\mathrm{B}=\mathrm{C} \square \mathrm{D}$ & \\
\hline $\begin{array}{l}\text { 2. ¿Sabes qué competencias transversales se abordan en la presente } \\
\text { asignatura? } \\
\text { A. Sí } \\
\text { B. No }\end{array}$ & $\nabla \mathrm{A} \backsim \mathrm{B} \backsim \mathrm{C} \square \mathrm{D}$ & \\
\hline
\end{tabular}




\begin{tabular}{|c|c|c|c|}
\hline 3. & $\begin{array}{l}\text { CT01 Comprensión e integración. En relación a la expresión de } \\
\text { ideas y generación de conclusiones, partiendo de distintos datos y } \\
\text { sus relaciones } \\
\text { A. Evalúo los datos y sus relaciones para llegar a conclusiones } \\
\text { adicionales a las expuestas en el paper. } \\
\text { B. Expreso ideas y genero conclusiones, partiendo de distintos } \\
\text { datos y sus relaciones } \\
\text { C. Me apoyo en datos, pero me cuesta tener en cuenta TODOS } \\
\text { D. ellos } \\
\text { Expongo mis ideas como opiniones, porque me cuesta } \\
\text { apoyarme en datos objetivos. }\end{array}$ & $\square \mathrm{A}\|\mathrm{B}\| \mathrm{C} \backsim \mathrm{D}$ & $\mathrm{A}=\mathrm{B}=\mathrm{c}$ \\
\hline 4. & $\begin{array}{l}\text { CT01 Comprensión e integración. En relación al establecimiento } \\
\text { de relaciones causa-efecto } \\
\text { A. Argumento correctamente las relaciones identificadas } \\
\text { B. Identifico correctamente relaciones causa-efecto } \\
\text { C. Algunas veces establezco relaciones de causalidad erróneas } \\
\text { D. Me cuesta identificar relaciones de causalidad }\end{array}$ & $\square \mathrm{A} \square \mathrm{B} \backsim \mathrm{C} \backsim \mathrm{D}$ & $\mathrm{B}=\mathrm{C}$ \\
\hline & $\begin{array}{l}\text { CT09 Pensamiento crítico } \\
\text { A. Selecciono con acierto el criterio a utilizar a la hora de } \\
\text { argumentar un juicio. Soy capaz de proponer alternativas } \\
\text { brillantes. } \\
\text { B. Argumento mis juicios y planteamientos, proponiendo } \\
\text { alguna alternativa lógica } \\
\text { C. Soy capaz de poner en duda alguna metodología o resultado } \\
\text { D. Formulo juicios sin conseguir apoyarme en criterios sólidos }\end{array}$ & $B=C \backsim D$ & D \\
\hline
\end{tabular}

Tabla 3. Encuesta inicial y final sobre el desarrollo del trabajo teórico de análisis de un artículo científico en la asignatura de Moldeling and simulation of bioelectric systems

\begin{tabular}{|c|c|c|c|}
\hline & Preguntas. Análisis del artículo científico n=19 & $\begin{array}{l}\text { Respuesta Inicial. } \\
\text { Avance. }\end{array}$ & $\begin{array}{l}\text { Respuesta Final. } \\
\text { Autoevaluación. }\end{array}$ \\
\hline & $\begin{array}{l}\text { ¿Hiciste una planificación inicial detallada de tareas y tiempo? } \\
\text { A. No } \\
\text { B. Sí, pero no detallada } \\
\text { C. Sí }\end{array}$ & $\square \mathrm{A} \square \mathrm{B} \square \mathrm{C} \square \mathrm{D}$ & \\
\hline 7. & $\begin{array}{l}\text { Valora tu grado de cumplimiento general del plan inicial de tareas } \\
\text { realizadas. } \\
\text { A. } \quad 0 \% \\
\text { B. } \quad 25 \% \\
\text { C. } \quad 50 \% \\
\text { D. } \quad 100 \%\end{array}$ & $\mathrm{~A} \backsim \mathrm{B} \backsim \mathrm{C} \square \mathrm{D}$ & $\because \mathrm{A} \backsim \mathrm{B} \backsim \mathrm{C} \backsim \mathrm{D}$ \\
\hline 8. & $\begin{array}{l}\text { ¿Has dedicado el tiempo previsto a las tareas desarrolladas hasta el } \\
\text { momento? He dedicado: } \\
\text { A. Menos tiempo } \\
\text { B. Más tiempo } \\
\text { C. El tiempo previsto }\end{array}$ & $\because A \backsim B \backsim C \backsim D$ & $\square \mathrm{A}=\mathrm{B}$ \\
\hline 9. & $\begin{array}{l}\text { Valora el trabajo en equipo de tu grupo } \\
\text { A. Participación desigual de los miembros y mala } \\
\text { compenetración } \\
\text { B. Participación equilibrada y compenetración mediocre } \\
\text { C. Participación equilibrada y buena compenetración } \\
\text { D. Otros: }\end{array}$ & $\square \mathrm{A} \square \mathrm{B} \square \mathrm{C} \square \mathrm{D}$ & $B \backsim C \backsim D$ \\
\hline 10. & $\begin{array}{l}\text { ¿Cuál es tu grado de satisfacción con el análisis del artículo? } \\
\text { A. Malo } \\
\text { B. Bueno } \\
\text { C. Muy bueno }\end{array}$ & & $\square \mathrm{A} \backsim \mathrm{B} \backsim \mathrm{C} \backsim \mathrm{D}$ \\
\hline 11. & $\begin{array}{l}\text { El análisis del artículo te ha ayudado a asimilar mejor los } \\
\text { contenidos de la asignatura: } \\
\text { A. No } \\
\text { B. Un poco } \\
\text { C. Bastante } \\
\text { D. Mucho }\end{array}$ & & $\square \mathrm{A} \backsim \mathrm{B} \backsim \mathrm{C} \square \mathrm{D}$ \\
\hline
\end{tabular}


Uso de la metodología de aprendizaje basada en proyectos para la adquisición de competencias transversales con niveles de dominio alto en asignaturas relacionadas con la Bioelectricidad

Tabla 4. Encuesta inicial y final sobre la adquisición de comptentecias transversales en el proyecto de simulación en la asignatura de Moldeling and simulation of bioelectric systems

\begin{tabular}{|c|c|c|c|}
\hline \multicolumn{2}{|r|}{ Preguntas Competencias. Proyecto de simulación n=17 } & Respuesta Inicial & \multirow[t]{2}{*}{ Respuesta Final } \\
\hline & $\begin{array}{l}\text { ¿Conoces el programa de competencias transversales de la UPV? } \\
\text { A. No } \\
\text { B. Muy poco } \\
\text { C. Bien } \\
\text { D. Muy bien }\end{array}$ & $\square \mathrm{A}=\mathrm{B} \backsim \mathrm{C}=\mathrm{D}$ & \\
\hline 2. & $\begin{array}{l}\text { ¿Sabes qué competencias transversales se abordan en la presente } \\
\text { asignatura? } \\
\text { A. Sí } \\
\text { B. No }\end{array}$ & $\square \mathrm{A} \square \mathrm{B} \backsim \mathrm{C} \square \mathrm{D}$ & \\
\hline 3. & $\begin{array}{l}\text { CT03 Análisis y resolución de problemas. En relación al análisis de las } \\
\text { causas y efectos de los problemas desde un enfoque global } \\
\text { A. Analizo las causas y efectos de los problemas con profundidad } \\
\text { B. Analizo las causas y efectos de los problemas correctamente } \\
\text { C. Analizo las causas y efectos de los problemas de manera } \\
\text { superficial } \\
\text { D. No logro analizar las causas y efectos de los problemas de } \\
\text { manera coherente }\end{array}$ & $\square=\mathrm{B}=\mathrm{C}$ & 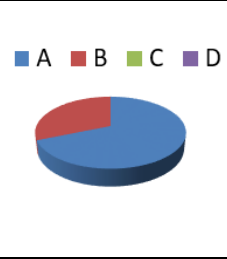 \\
\hline 4. & $\begin{array}{l}\text { CT03 Análisis y resolución de problemas. En relación a la evaluación } \\
\text { de las posibles soluciones según su viabilidad científico-técnica y } \\
\text { dificultad de implementación } \\
\text { A. Analizo la viabilidad de las soluciones, describiendo las ventajas } \\
\text { e inconvenientes de su aplicación práctica y tomando decisiones } \\
\text { en consecuencia } \\
\text { B. Analizo la viabilidad de las soluciones describiendo sus ventajas } \\
\text { e inconvenientes de su aplicación práctica pero me cuesta tomar } \\
\text { decisiones en consecuencia } \\
\text { C. Describo ventajas e inconvenientes de las posibles soluciones } \\
\text { pero sin justificar del todo cómo afectan a la viabilidad de la } \\
\text { solución propuesta } \\
\text { D. Presento soluciones adecuadas al problema sin discutir en } \\
\text { profundidad su viabilidad o implicaciones }\end{array}$ & D & D \\
\hline 5. & $\begin{array}{l}\text { CT03 Análisis y resolución de problemas. En relación a la } \\
\text { organización de una manera sistemática el trabajo para la toma de } \\
\text { decisiones (en grupo) } \\
\text { A. Distingo los diferentes procesos a realizar para resolver el } \\
\text { problema, establezco una secuencia de tareas, señalo las } \\
\text { implicaciones entre tareas y defino indicadores para la toma de } \\
\text { decisiones }\end{array}$ & $\square \mathrm{A}$ & $\square \mathrm{A}$ \\
\hline & $\begin{array}{l}\text { CT04 Innovación, creatividad y emprendimiento. En relación a la } \\
\text { adopción de enfoques creativos en el contenido y modo de realización: } \\
\text { A. Adopto enfoques originales, genero nuevas ideas divergentes a } \\
\text { partir de perspectivas diferentes } \\
\text { B. y aporta creatividad en lo que hago, mejorando sistemas, } \\
\text { procedimientos y procesos } \\
\text { C. Adopto enfoques adecuados al contenido de la situación y un } \\
\text { correcto planteamiento del modo de realización } \\
\text { D. Me cuesta todavía proponer ideas y enfoques que se adapten al } \\
\text { problema y/o me cuesta especificar correctamente el modo de } \\
\text { ejecución }\end{array}$ & $\square \mathrm{A} \square \mathrm{B} \square \mathrm{C} \square \mathrm{D}$ & $\square \mathrm{A} \square \mathrm{B}$ \\
\hline
\end{tabular}


Tabla 5. Encuesta inicial y final sobre el desarrollo del proyecto de simulación en la asignatura de Moldeling and simulation of bioelectric systems

\begin{tabular}{|c|c|c|}
\hline Preguntas. Análisis del proyecto de simulación n=17 & $\begin{array}{l}\text { Respuesta Inicial. } \\
\text { Avance. }\end{array}$ & $\begin{array}{l}\text { Respuesta Final. } \\
\text { Autoevaluación. }\end{array}$ \\
\hline $\begin{array}{l}\text { 7. ¿Hiciste una planifícación inicial detallada de tareas y tiempo? } \\
\text { A. No } \\
\text { B. Sí, pero no detallada } \\
\text { C. Sí }\end{array}$ & & \\
\hline $\begin{array}{l}\text { 8. Valora tu grado de cumplimiento general del plan inicial de tareas } \\
\text { realizadas. } \\
\text { E. } \quad 0 \% \\
\text { F. } \quad 25 \% \\
\text { G. } \quad 50 \% \\
\text { H. } \quad 100 \% \\
\end{array}$ & $\mathrm{~A} \backsim \mathrm{B} \backsim \mathrm{C} \backsim \mathrm{D}$ & $C \square D$ \\
\hline $\begin{array}{l}\text { 9. ¿Has dedicado el tiempo previsto a las tareas desarrolladas hasta el } \\
\text { momento? He dedicado: } \\
\text { A. Menos tiempo } \\
\text { B. Más tiempo } \\
\text { C. El tiempo previsto }\end{array}$ & $\because \mathrm{A} \square \mathrm{B} \square \mathrm{C} \square \mathrm{D}$ & $C \backsim D$ \\
\hline $\begin{array}{l}\text { 10. Valora el trabajo en equipo de tu grupo } \\
\text { A. Participación desigual de los miembros y mala compenetración } \\
\text { B. Participación equilibrada y compenetración mediocre } \\
\text { C. Participación equilibrada y buena compenetración } \\
\text { D. Otros: }\end{array}$ & $\square \mathrm{A} \backsim \mathrm{B} \backsim \mathrm{C} \backsim \mathrm{D}$ & 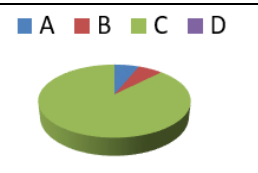 \\
\hline $\begin{array}{l}\text { 11. ¿Cuál es tu grado de satisfacción con el análisis del artículo? } \\
\text { A. Malo } \\
\text { B. Bueno } \\
\text { C. Muy bueno }\end{array}$ & & $\mathrm{A}=\mathrm{B}=\mathrm{C} \square \mathrm{D}$ \\
\hline $\begin{array}{l}\text { 12. El proyecto te ha ayudado a asimilar mejor los contenidos de la } \\
\text { asignatura: } \\
\text { A. No } \\
\text { B. Un poco } \\
\text { C. Bastante } \\
\text { D. Mucho }\end{array}$ & & $\because \mathrm{A} \backsim \mathrm{B} \backsim \mathrm{C} \backsim \mathrm{D}$ \\
\hline
\end{tabular}

Según las encuestas realizadas a los alumnos de la asignatura de Bioelectrónica (tabla 6), éstos son inicialmente poco conocedores de las competencias transversales que se trabajan en la asignatura, pero evidentemente al finalizar la asignatura consideran que son buenos conocedores. Además, al finalizar la asignatura reconocen haber alcanzado un nivel alto en las competencias CT05 у CT08. Concretamente, los alumnos han alcanzado un alto nivel en diseño y proyecto, progresando a lo largo del curso en el planteamiento de objetivos y planificación, así como en el análisis de resultados. En cuanto a la comunicación oral y escrita, los alumnos lo han trabajado de manera intensa en el desarrollo de los distintos trabajos teóricos y prácticos y son conscientes de su progreso en este campo. Al finalizar la asignatura consideran que han alcanzado un nivel alto en el desarrollo de sus exposiciones orales y en la estructura, fondo y forma de sus informes escritos.

En cuanto al tiempo dedicado a los trabajos de la asignatura (tabla 7), en general consideran que han dedicado el tiempo previsto, pero quizás algo más de tiempo a los trabajos prácticos, donde el trabajo en equipo no ha sido tan bien valorado como en los trabajos teóricos. El grado de satisfacción general ha sido muy bueno y los alumnos consideran que la realización de los trabajos propuestos y las metodologías empleadas les han ayudado mucho a adquirir las competencias trabajadas y afianzar los contenidos de la asignatura. 
Uso de la metodología de aprendizaje basada en proyectos para la adquisición de competencias transversales con niveles de dominio alto en asignaturas relacionadas con la Bioelectricidad

Tabla 6. Encuesta inicial y final sobre la adquisicón de competencias transversales durante el desarrollo de los trabajos prácticos y teóricos en la asignatura de Bioelectrónica.

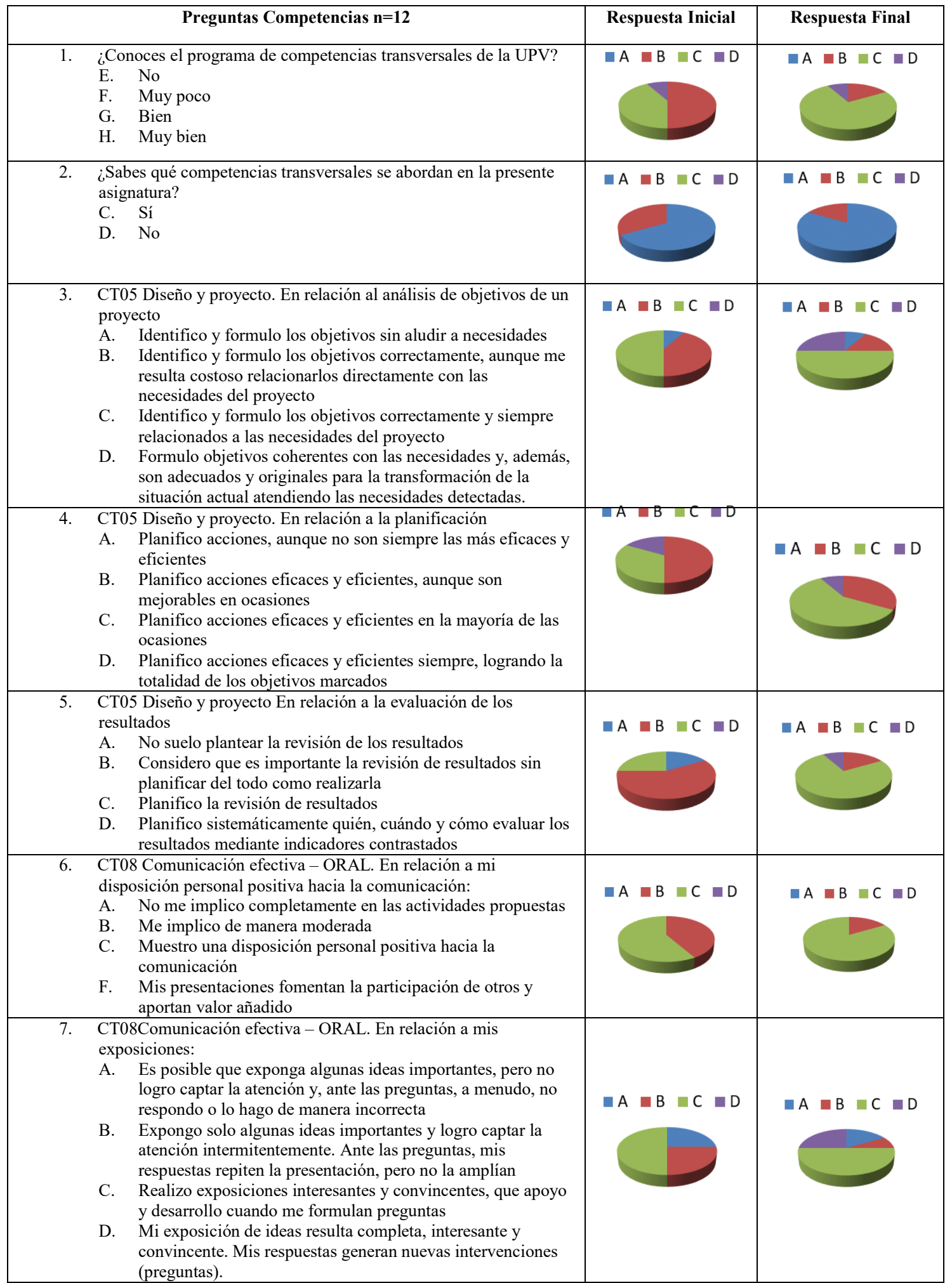




\begin{tabular}{|c|c|c|c|}
\hline 8. & $\begin{array}{l}\text { CT08 Comunicación efectiva-ORAL. En relación a la } \\
\text { preparación de la exposición: } \\
\text { A. La exposición no está siempre debidamente estructurada y/o } \\
\text { no se ajusta al tiempo establecido. } \\
\text { B. La exposición está organizada, pero hay demasiada o escasa } \\
\text { información y/o no se ajusta al tiempo establecido } \\
\text { C. La audiencia puede captar claramente la eficaz estructura del } \\
\text { E. Lantenido y la exposición se ajusta al tiempo establecido } \\
\text { La selección y organización del contenido y su ilustración } \\
\text { (ejemplos, analogías...) son coherentes y la exposición se } \\
\text { ajusta al tiempo establecido }\end{array}$ & $\because A \backsim B \backsim C \backsim D$ & $C \backsim D$ \\
\hline 9. & $\begin{array}{l}\text { CT08 Comunicación efectiva-ORAL. En relación a mi expresión } \\
\text { oral: } \\
\text { A. En general no realizo un buen uso del lenguaje y/o no utilizo } \\
\text { B. la terminología específica de la materia } \\
\text { La presentación no es fluida (lenguaje limitado) y utilizo } \\
\text { escasa terminología específica o lo hago inapropiadamente } \\
\text { C. Respeto las normas lingüísticas y me expreso de manera } \\
\text { correcta, utilizando de modo pertinente la terminología } \\
\text { específica de la materia } \\
\text { D. La exposición se adapta a la audiencia, haciendo uso de un } \\
\text { lenguaje y de una terminología específicos, apropiados, } \\
\text { precisos y ricos }\end{array}$ & $C \backsim D$ & $\square \mathrm{A}$ \\
\hline 10. & $\begin{array}{l}\text { CT08 Comunicación efectiva - ORAL. En relación a la } \\
\text { comunicación no verbal: } \\
\text { A. Mi lenguaje no verbal no respalda y/o distrae mi discurso } \\
\text { verbal } \\
\text { B. Mi lenguaje no verbal muestra alguna/s deficiencia/s } \\
\text { C. Mi lenguaje no verbal es coherente con el verbal } \\
\text { D. Mi lenguaje no verbal refuerza el verbal: gestos firmes, ritmo } \\
\text { variado y entonación intencional }\end{array}$ & $\square \mathrm{A} \square \mathrm{B}$ & $C \backsim D$ \\
\hline 11. & $\begin{array}{l}\text { CT08 Comunicación efectiva - ESCRITA. En relación al } \\
\text { desarrollo del tema: } \\
\text { A. Uso párrafos ajenos sin citar SIEMPRE la fuente (plagio, } \\
\text { "cortar y pegar") y/o presento un desarrollo incompleto del } \\
\text { tema } \\
\text { B. El desarrollo del tema es completo, pero con escasa } \\
\text { elaboración personal } \\
\text { C. El desarrollo del tema es original y completo: fundamento } \\
\text { mis reflexiones a partir de fuentes variadas, actualizadas y } \\
\text { relevantes } \\
\text { D. Destaco mi riqueza argumentativa y utilizo fuentes variadas, } \\
\text { actualizadas y relevantes para apoyar o refutar mis } \\
\text { planteamientos. }\end{array}$ & $\square \mathrm{A} \square \mathrm{B}$ & \\
\hline 12. & $\begin{array}{l}\text { CT08 Comunicación efectiva - ESCRITA. En relación a la } \\
\text { estructura del documento: } \\
\text { A. No utilizo numeraciones y/o no sigo siempre las } \\
\text { convenciones habituales de mi disciplina } \\
\text { B. Uso de forma inadecuada distintos tipos de numeración y/o } \\
\text { no siempre sigo las convenciones habituales de mi disciplina } \\
\text { C. Me adapto a las convenciones habituales de mi disciplina y } \\
\text { facilito la comprensión mediante un texto bien estructurado } \\
\text { que combina subepígrafes, numeraciones, etc. } \\
\text { D. Destaco por mi estilo personal a la hora de estructurar el } \\
\text { texto y mi capacidad de manejo y adaptación a los diferentes } \\
\text { usos y convenciones habituales de mi disciplina }\end{array}$ & $\square \mathrm{A}$ & D \\
\hline 13. & $\begin{array}{l}\text { CT08 Comunicación efectiva-ESCRITA. En relación al uso } \\
\text { correcto de la gramática: } \\
\text { A. Debería mejorar el uso de los signos de puntuación, para } \\
\text { facilitar una fácil comprensión del texto } \\
\text { B. Pese a que presento un texto gramaticalmente correcto, su } \\
\text { lectura resulta aburrida, monótona y/o poco fluida } \\
\text { C. Mi corrección gramatical ayuda a transmitir las ideas de } \\
\text { manera clara y comprensible } \\
\text { D. Mis construcciones personales se adaptan específicamente al } \\
\text { lector y mi estilo resulta fluido y ameno, más allá de claro y } \\
\text { comprensible }\end{array}$ & $=\mathrm{D}$ & $\square=\mathrm{B}$ \\
\hline
\end{tabular}


Uso de la metodología de aprendizaje basada en proyectos para la adquisición de competencias transversales con niveles de dominio alto en asignaturas relacionadas con la Bioelectricidad

\begin{tabular}{|c|c|c|c|}
\hline & $\begin{array}{l}\text { CT08 Comunicación efectiva-ESCRITA. En relación al uso de } \\
\text { lenguaje específico de mi disciplina: } \\
\text { A. No utilizo el lenguaje específico de la disciplina de forma del } \\
\text { todo apropiada } \\
\text { B. Utilizo el lenguaje específico de la disciplina ocasionalmente } \\
\text { C. Utilizo el lenguaje específico de la disciplina de forma } \\
\text { apropiada } \\
\text { D. Destaco por un estilo personal que se plasma en un repertorio } \\
\text { lingüístico rico, apropiado y diverso. }\end{array}$ & $\square \mathrm{A} \square \mathrm{B} \square \mathrm{C} \square \mathrm{D}$ & $\square A \square=C \square D$ \\
\hline 15. & $\begin{array}{l}\text { CT08 Comunicación efectiva - ESCRITA. En relación al uso de } \\
\text { los recursos de apoyo: } \\
\text { A. Los recursos de apoyo son limitados y/o de escasa calidad } \\
\text { B. Uso recursos de apoyo apropiados, pero no les saco el mejor } \\
\text { partido ni se adaptan del todo al tipo de texto ni al público } \\
\text { lector } \\
\text { C. Utilizo los recursos de apoyo más apropiados para mantener } \\
\text { D. Los interés e incitar a la reflexión } \\
\text { D. los de apoyo son variados, originales y/o creativos } \\
\text { y aportan valor añadido al escrito, propiciando la reflexión } \\
\text { del lector }\end{array}$ & $C \backsim D$ & $B \backsim C \backsim D$ \\
\hline
\end{tabular}

Tabla 7. Encuesta sobre el desarrollo de los trabajos prácticos y teóricos en la asignatura de Bioelectrónica.

\begin{tabular}{|c|c|c|}
\hline Preguntas Trabajos $n=12$ & Trabajo Teórico & Trabajo Práctico \\
\hline $\begin{array}{l}\text { 16. ¿Has dedicado el tiempo previsto a las tareas desarrolladas hasta el } \\
\text { momento? He dedicado: } \\
\text { A. Menos tiempo } \\
\text { B. Más tiempo } \\
\text { C. El tiempo previsto }\end{array}$ & $\mathrm{C} \backsim \mathrm{D}$ & Prácticos \\
\hline $\begin{array}{l}\text { 17. Indica el número de horas aproximadas dedicadas a cada uno de } \\
\text { los trabajos teóricos/prácticos: } \\
\text { A. } 5-10 \\
\text { B. } 10-20 \\
\text { C. } 20-30 \\
\text { D. } \quad 30-40\end{array}$ & $\square \mathrm{A} \square \mathrm{B} \square \mathrm{C}$ & $\mathrm{A}$ \\
\hline $\begin{array}{l}\text { 18. Valora el trabajo en equipo de tu grupo } \\
\text { A. Participación desigual de los miembros y mala } \\
\text { compenetración } \\
\text { B. Participación equilibrada y compenetración mediocre } \\
\text { C. Participación equilibrada y buena compenetración } \\
\text { D. Otros: }\end{array}$ & $C \backsim D$ & $\mathrm{~B} \backsim \mathrm{C} \backsim \mathrm{D}$ \\
\hline $\begin{array}{l}\text { 19. ¿Cuál es tu grado de satisfacción con los trabajos } \\
\text { teóricos/prácticos? } \\
\text { A. Malo } \\
\text { B. Bueno } \\
\text { C. Muy bueno }\end{array}$ & $=\mathrm{D}$ & $C \backsim D$ \\
\hline $\begin{array}{l}\text { 20. Los trabajos teóricos te han ayudado a asimilar mejor los } \\
\text { contenidos de la asignatura: } \\
\text { A. No } \\
\text { B. Un poco } \\
\text { C. Bastante } \\
\text { D. Mucho }\end{array}$ & $D$ & $\square \mathrm{A}=\mathrm{B} \square \mathrm{C} \square \mathrm{D}$ \\
\hline $\begin{array}{l}\text { 21. ¿Te ha parecido una buena metodología de aprendizaje el póster } \\
\text { científico/diario de laboratorio? } \\
\text { A. No } \\
\text { B. Un poco } \\
\text { C. Bastante } \\
\text { D. Mucho }\end{array}$ & $\because \mathrm{A} \backsim \mathrm{B} \backsim \mathrm{C} \square \mathrm{D}$ & $\square \mathrm{A} \square \mathrm{B} \square \mathrm{C} \square \mathrm{D}$ \\
\hline \multicolumn{3}{|l|}{$\begin{array}{l}\text { ¿Cuál es la principal dificultad que has encontrado en los trabajos } \\
\text { teóricos/prácticos? }\end{array}$} \\
\hline stas de mejora para lo & & \\
\hline
\end{tabular}

(cc) BY-NC-ND 2020, Universitat Politècnica de València

Congreso In-Red (2020) 
Con respecto al tiempo dedicado de los profesores participantes, puede afirmarse que ha correspondido con la planificación inicial y el desarrollo de los trabajos es viable en las 2 asignaturas. La principal inversión temporal ha sido para la preparación del material, enunciados, plantillas y rúbricas. El seguimiento del trabajo no ha supuesto una gran inversión puesto que los grupos no son demasiado numerosos (20 y 30 alumnos en cada una de las asignaturas) y son alumnos con gran independencia al ser de últimos cursos.

El diseño de rúbricas detalladas permitieron evaluar el nivel de dominio de las competencias aplicadas en el transcurso del aprendizaje basado en proyectos. Estas rúbricas fueron la base de la evaluación de los proyectos realizados.

La documentación exigida a los alumnos (planificación, reportes, presentaciones, etc.) también han servido de evidencia para evaluar el logro de los objetivos.

Los resultados de evaluación han sido los que se muestran en la tablas 8 y 9 para las 2 asignaturas respectivamente.

Tabla 8 y 9. Resultados de evaluación en la asignatura de Moldeling and simulation of bioelectric systems(izquierda) y Bioelectrónica (derecha)

\begin{tabular}{|c|c|}
\hline Competencia & Resultados \\
\hline CT01 & A. Excelente $100 \%$ \\
\hline CT03 & $\begin{array}{l}\text { A. Excelente } 68 \% \\
\text { B. Adecuado } 32 \%\end{array}$ \\
\hline CT04 & $\begin{array}{l}\text { A. Excelente } 54 \% \\
\text { B. Adecuado } 46 \%\end{array}$ \\
\hline CT09 & $\begin{array}{l}\text { A. Excelente } 36 \% \\
\text { B. Adecuado } 64 \%\end{array}$ \\
\hline
\end{tabular}

\begin{tabular}{|c|c|}
\hline Competencia & Resultados \\
\hline CT05 & A. Excelente $53 \%$ \\
& B. Adecuado $47 \%$ \\
\hline CT08 & A. Excelente $60 \%$ \\
& B. Adecuado $40 \%$ \\
\hline
\end{tabular}

\section{Conclusiones}

El diseño y aplicación de la metodología de aprendizaje basada en proyectos en las asignaturas de Bioelectrónica y Modeling and simulation of bioelectric systems ha permitido la integración de las competencias transversales "Diseño y proyecto" (CT05), "Comunicación efectiva" (CT08), "Compresión e integración" (CT01), "Análisis y resolución de problemas" (CT03), "Innovación, creatividad y emprendimiento" (CT04) y "Pensamiento crítico" (CT09), con altos niveles de dominio. Se ha aplicado esta metodología a lo largo del curso 2019-2020 en dichas asignaturas pertenecientes al Grado de Ingeniería Electrónica Industrial y Automática y el Máster de Ingeniería Biomédica, respectivamente de la Universitat Politècnica de València. La valoración del alumnado y del profesorado ha sido muy positiva y la adquisición de competencias transversales ha sido muy satisfactoria.

\section{Referencias}

FERNÁNDEZ MARCH, A (2006) "Metodologías activas para la formación de competencias" Educatio siglo XXI, vol. 24, pp 35-56.

PRATS-BOLUDA, G,. YE LIN, Y, TRENOR, B (2016). "Análisis del uso del póster científico y de la revisión por pares como herramienta desarrollo de la competencia comunicación efectiva en estudiantes de grado en ingeniería". Congreso Nacional de Innovación Educativa y Docencia en Red (1 - 12). Valencia, España: Editorial UPV.

PRATS-BOLUDA, G, YE LIN, Y, BOSCH ROIG, I, MARTÍNEZ DE JUAN, JL (2017). "Análisis del uso de la metodología aprendizaje basado en problemas como Herramienta de desarrollo de competencias en estudiantes de 
Uso de la metodología de aprendizaje basada en proyectos para la adquisición de competencias transversales con niveles de dominio alto en asignaturas relacionadas con la Bioelectricidad

grado de ingeniería" 5th International Conference on Innovation, Documentation and Teaching Technologies (INNODOCT 2017). (1 - 11). Valencia, Spain: Editorial Universitat Politècnica de València.

TIPPELT, R, LINDEMANN, H. (2001). El método de Proyectos. El Salvador, München Berlin.

TRENOR, B, PRATS-BOLUDA, G, YE LIN, Y (2017). “Aplicación de la Clase Inversa en la Enseñanza de la Electrónica Analógica en un Grupo de Alto Rendimiento Académico" Congreso Nacional de Innovación Educativa y Docencia en Red (IN-RED 2017). (1 - 12). Valencia, Spain: Editorial UPV.

YE LIN, Y, PRATS-BOLUDA, G, GARCIA CASADO, FJ, GUIJARRO ESTELLES, E, MARTÍNEZ DE JUAN, JL (2017). "Análisis del empleo de la metodología aprendizaje basado en proyectos como herramienta de desarrollo y evaluación de múltiples competencias transversales. Aplicación en grupos numerosos de asignaturas en la rama de ingeniería" Congreso Nacional de Innovación Educativa y Docencia en Red (IN-RED 2017). (1 - 15). Valencia, Spain: Editorial UPV.

YE LIN, Y, PRATS-BOLUDA, G, GARCIA-CASADO, J, MARTINEZ-MILLANA, A, GUIJARRO ESTELLES, E, MARTÍNEZ DE JUAN, JL (2018). "Desarrollo e implantación de un sistema de evaluación objetiva del aprendizaje individual en trabajos grupales en grupos numerosos de asignaturas de ingeniería". IV Congreso Nacional de Innovación Educativa y Docencia en Red (IN-RED 2018). Valencia, España: Editorial Universitat Politècnica de València.

YE-LIN, Y, PRATS-BOLUDA, G, GARCIA-CASADO, J, GUIJARRO ESTELLES,E, MARTINEZ-DE-JUAN, JL (2019). "Diseño, aplicación y valoración de actividades destinadas al trabajo y evaluación de múltiples competencias transversales en grupos numerosos de máster en ingeniería, empleando el aprendizaje basado en proyectos" Educatio S. XX1. Aceptado, en proceso de publicación.

ZABALZA M.Á (2011) "Metodología docente". REDU-Revista de docencia Universitaria, 9(3) pp75-89. http://dx.doi.org/10.4995/redu.2011.6150. 